\title{
Lenalidomide maintenance in myeloma
}

\author{
Alessandro Gozzetti, Marzia Defina and Monica Bocchia
}

We strongly agree with Rajkumar (Haematological cancer: Lenalidomide maintenance-perils of a premature denouement. Nat. Rev. Clin. Oncol. 9, $372-374 ; 2012)^{1}$ that it is premature to recommend lenalidomide maintenance to all patients with myeloma, but we would also like to underline other aspects of the problem not discussed in the recent commentary article.

It is our assertion that not every patient with myeloma needs maintenance therapy. In addition to considering the risk-benefit ratio for a patient during maintenance therapy, we are convinced that the wellknown concept of disease plateau-which was established decades before the novelagent era in myeloma ${ }^{2}$-is also applicable in the setting of maintenance therapy. Furthermore, a small percentage of patients (15\%, but this could improve with novel agents used in the pre-transplant setting) after autologous stem-cell transplantation (ASCT) achieve disease-free survival or progression-free survival of longer than 10 years. ${ }^{3}$ For those patients, any maintenance therapy is useless or, even worse, harmful. When considering maintenance therapy, especially after ASCT, the primary aim should be a survival benefit for the patients. Other considerations should include patient quality of life and cost effectiveness.

To assess the value of maintenance therapy we should define the meaning of maintenance therapy as compared with consolidation therapy-if measurable disease is present, we should define a given therapy as 'consolidation'. This aspect is very important, because the advantages in overall survival and progression-free survival seen in patients receiving maintenance therapy in the trial published by McCarthy et al. ${ }^{4}$ was in patients who had not achieved a complete response on their previous therapy. The presence of measurable disease in these patients was not commented on by Rajkumar ${ }^{1}$ in his commentary article, and compares with the patients with complete response in the trials conducted by Palumbo et al. ${ }^{5}$ and Attal et al. ${ }^{6}$ In both of these trials, an overall survival benefit was not associated with lenalidomide maintenance therapy, although a progression-free survival benefit was seen.,

It seems that there is no doubt that lenalidomide is useful in patients with residual disease ${ }^{7}$ (that is, as consolidation therapy); however, we have some concerns that lenalidomide treatment can be useful in patients who have already achieved complete response. With the advent of new agents, achieving a complete response after ASCT is now more likely than in the past, ${ }^{8}$ and we should better understand in future trials those patients who will benefit from lenalidomide or bortezomib maintenance therapy and those who will not. For these reasons we believe that lenalidomide use should be rationally planned.

Division of Hematology and Transplants, University of Siena, Policlinico "Santa Maria alle Scotte", Viale Bracci 16, 53100 Siena, Italy (A. Gozzetti, M. Defina, M. Bocchia).
Correspondence to: A. Gozzetti gozzetti@unisi.it

Acknowledgements

The authors would like to thank Siena A. I. L. (Italian Association against Leukemia, Lymphoma and Myeloma, Siena District, Italy), and all the doctors, nurses, and laboratory technicians working at the Division of Hematology and Transplants, AOUS, Policlinico Le Scotte, Siena for their daily assistance in patient care.

Competing interests

The authors declare no competing interests.

1. Rajkumar, S. V. Haematological cancer: Lenalidomide maintenance-perils of a premature denouement. Nat. Rev. Clin. Oncol. 9, 372-374 (2012).

2. Durie, B. G., Russell, D. H. \& Salmon, S. E. Reappraisal of plateau phase in myeloma. Lancet 2, 65-68 (1980).

3. Martinez Lopez, J. et al. Long-term prognostic significance of response in multiple myeloma after stem cell transplantation. Blood 118, 529-534 (2011).

4. McCarthy, P. L. et al. Lenalidomide after stem cell transplantation for multiple myeloma. N. Engl. J. Med. 366, 1770-1781 (2012).

5. Palumbo, A. et al. Continuous lenalidomide treatment for newly diagnosed multiple myeloma. N. Engl. J. Med. 366, 1759-1769 (2012).

6. Attal, M. et al. Lenalidomide maintenance after stem cell transplantation for multiple myeloma. N. Engl. J. Med. 366, 1782-1791 (2012).

7. Dimopoulos, M. A. et al. Long-term follow-up on overall survival from the MM-009 and MM-010 phase III trials of lenalidomide plus dexamethasone in patients with relapsed or refractory multiple myeloma. Leukemia $\mathbf{2 3}$, 2147-2152 (2009).

8. Cavo, M. et al. Bortezomib with thalidomide plus dexamethasone compared with thalidomide plus dexamethasone as induction therapy before, and consolidation therapy after, double autologous stem-cell transplantation in newly diagnosed multiple myeloma: a randomised phase 3 study. Lancet 376, 2075-2085 (2010). 